\title{
Phenolic compounds in cereal grains and effects of processing on their composition and bioactivities: a review
}

\author{
Fereidoon Shahidi*, Renan Danielski and Chiaki Ikeda
}

Department of Biochemistry, Memorial University of Newfoundland, St. John's, NL, Canada A1B 3X9

${ }^{*}$ Corresponding author: Department of Biochemistry, Memorial University of Newfoundland, St. John's, NL, Canada A1B 3X9. E-mail: fshahidi@mun.ca

DOI: $10.31665 / J F B .2021 .15281$

Received: September 27, 2021; Revised received \& accepted: September 29, 2021

Citation: Shahidi, F., Danielski, R., and Ikeda, C. (2021). Phenolic compounds in cereal grains and effects of processing on their composition and bioactivities: a review. J. Food Bioact. 15: 39-50.

\begin{abstract}
Cereals are a staple food in the diets of many populations globally. Besides their nutritive function in food, they are also rich in various groups of bioactive compounds, especially polyphenols. Wheat, rice, barley, rye, oat, maize, millet, sorghum, and other cereal grains present a great variety of phenolic acids, flavonoids, proanthocyanidins, alkylresorcinols, and lignans, which can be affected in many ways by the post-harvest treatments and further processing of these feedstocks. This review discusses up-to-date studies about the effects of common cereal processing techniques on their phenolic composition, biological activities, and bioefficiency. Generally, mild thermal and high-pressure treatments enhance cereals' phenolic composition by releasing the insoluble-bound fraction, which increases their bioaccessibility. On the other hand, processes involving extreme temperature conditions and removal of the grains' outer layers may drastically reduce the phenolic content. Therefore, it is imperative to optimize the processing conditions of cereals, so their health-promoting benefits are preserved.
\end{abstract}

Keywords: Polyphenols; Antioxidant activity; Post-harvest treatment; Insoluble-bound; Bioaccessibility.

\section{Introduction}

Cereal cultivation has been a crucial factor for the development of many countries due to its economic and nutritional importance. As staple foods, they are able to provide a significant portion of energy and dietary needs. Cereals are rich in protein, selected micronutrients, and even antinutrients. Maize, rice, wheat, barley, sorghum, millets, oat, and rye are among the most cultivated cereals globally, playing a vital role in the economy of both developed and developing countries (Raheem et al., 2021). However, the importance of cereals extends beyond their essential nutrients. The consumption of whole grains (containing endosperm, germ, and bran) has been associated with a decreased incidence of cardiovascular diseases, some types of cancer, type 2 diabetes, and obesity, among others (Shahidi, 2009). These effects are due to the action of bioactive compounds present in such foods (Shahidi et al., 2019).

The most prominent bioactive classes of compounds found in cereals include phenolics, carotenoids, phytosterols, beta-glucans, and gamma-oryzanol. In raw cereals, their amount and profile vary according to their location in the grain. Usually, refined cereals possess an underwhelming bioactive composition compared to whole grains due to the removal of germ and bran, where several bioactives are located. The mechanism through which these compounds exert their beneficial effects varies according to their type, structure, occurrence in the matrix, and other conditions. Nevertheless, a number of bioactive substances have in common their capacity to act as antioxidants, dampening oxidative stress in the body, thus protecting the macromolecules (e.g., lipids, proteins, DNA) against damage that can lead to the development of chronic diseases. This has been deemed one of their most appealing features, helping the promotion of consumption of bioactive-rich foods and their capacity to serve as ingredients for nutraceutical and functional food development (Naczk and Shahidi, 2006).

The susceptibility of bioactive compounds in cereals is influenced by temperature and $\mathrm{pH}$ variations, exposure to light and ox- 
Table 1. Chemical composition of the major cereal grains produced worldwide (whole grain)

\begin{tabular}{llllllll}
\hline & Wheat & Rye & Maize & Barley & Oats & Rice & Millet \\
\hline Moisture (g/100g) & 12.6 & 13.6 & 11.3 & 12.1 & 13.1 & 13.0 & 12.0 \\
Protein (g/100g) & 11.3 & 9.4 & 8.8 & 11.1 & 10.8 & 7.7 & 10.5 \\
Lipids (g/100g) & 1.8 & 1.7 & 3.8 & 2.1 & 7.2 & 2.2 & 3.9 \\
Available carbohydrates (g/100g) & 59.4 & 60.3 & 65.0 & 62.7 & 56.2 & 73.7 & 68.2 \\
Fiber (g/100g) & 13.2 & 13.1 & 9.8 & 9.7 & 9.8 & 2.2 & 3.8 \\
Minerals (g/100g) & 1.7 & 1.9 & 1.3 & 2.3 & 2.9 & 1.2 & 1.6 \\
Thiamine (B1) (mg/kg) & 4.6 & 3.7 & 3.6 & 4.3 & 6.7 & 4.1 & 4.3 \\
Riboflavin (B2) (mg/kg) & 0.9 & 1.7 & 2.0 & 1.8 & 1.7 & 0.9 & 1.1 \\
Nicotinamide (mg/kg) & 51.0 & 18.0 & 15.0 & 48.0 & 24.0 & 52.0 & 18.0 \\
Panthothenic acid (mg/kg) & 12.0 & 15.0 & 6.5 & 6.8 & 7.1 & 17.0 & 14.0 \\
Vitamin B6 (mg/kg) & 2.7 & 2.3 & 4.0 & 5.6 & 9.6 & 2.8 & 5.2 \\
Folic acid (mg/kg) & 0.9 & 1.4 & 0.3 & 0.7 & 0.3 & 0.2 & 0.4 \\
Tocols (mg/kg) & 41.0 & 40.0 & 66.0 & 22.0 & 18.0 & 19.0 \\
\hline
\end{tabular}

Adapted from Koehler and Wieser (2013).

ygen, and enzymatic reactions. Such factors can compromise their ability to serve as antioxidants and exert their biological activities (Chawda et al., 2017). Moreover, exposure of bioactives to the aforementioned environmental factors is common for cereal grains and cereal products due to post-harvest treatment and storage. At this stage, cereals undergo a series of processing steps designed to clean, sort, and remove inedible portions (primary processing) and can even be transformed into more appealing products (secondary processing), being subjected to more extreme processes such as frying and extrusion (Food and Agriculture Organization, 2021). Therefore, it is imperative to analyze how the major bioactives found in cereals are affected by grain processing.

This review discusses up-to-date information on the transformations of cereal grains upon processing and the effects of commonly used unit operations on phenolic compounds, one the most relevant groups of bioactive substances encountered in highly produced cereals.

\section{Cereal grains: economic and nutritional importance}

Cultivation of cereal grains drives the economy of several territories worldwide. The Food and Agriculture Organization (FAO) of the United Nations estimates that around $70 \%$ of the world's farmland is used to cultivate cereal grains. Approximately $45 \%$ of the world's economically active population is engaged in agriculturerelated activities, primarily planting and harvesting cereal grains (Serna-Saldivar, 2016). Data from the 2020/2021 harvest (Statista, 2021 ) shows that corn (maize) is the most produced cereal in the world, reaching 1.12 billion metric tons, followed by wheat $(775.8$ million metric tons $(\mathrm{Mt}))$, milled rice $(505 \mathrm{Mt})$, barley $(159.74 \mathrm{Mt})$, sorghum $(62.05 \mathrm{Mt})$, oats $(25.53 \mathrm{Mt})$, and rye $(14.3 \mathrm{Mt})$. China is the largest cereal producer with over $612 \mathrm{Mt}$ harvested in 2018, followed by the United States with $467 \mathrm{Mt}$, and India with 318 Mt. Most of this production is directed to animal feed and ethanol manufacture, with human consumption receiving the smallest fraction. According to Food and Agriculture Organization (2020), cereal production is expected to grow by $375 \mathrm{Mt}$ in 2029 . This expansion is due to the development of better agricultural practices and advances in biotechnology.

From a nutritional standpoint, the availability of cereals is important for meeting the global food demand since it is a cost-effective and nutritious food option, especially when considering the population of developing countries (Raheem et al., 2021). Cereals are botanically classified as grasses belonging to the monocot family Poaceae. According to climate demands, cereals can be either warm-season or cool-season. Warm-season cereals encompass those grown year-round in tropical climates and during the frostfree season in temperate climates. Examples include corn, rice, sorghum, and millet. On the other hand, cool-season cereals are grown in moderate climates and include wheat, barley, oats, and rye (Koehler and Wieser, 2013). Anatomically, cereal grains are composed of bran, germ, and endosperm. The endosperm is the inner layer, making up most of the grain, and it is rich in starch and proteins. The germ is a small nutrient-rich core containing vitamins, fatty acids, and bioactive compounds. The bran is the outer layer involving the endosperm, a source of fiber, B vitamins, and trace minerals. The husk is combined with the bran in selected species, such as oats, barley, and rice (Sidhu et al., 2007).

Chemically, cereal grains are mainly composed of carbohydrates $(56-74 \%)$, especially the starch fraction deposited in the endosperm, with fiber (mainly in the bran) representing 2-13\% (Koehler and Wieser, 2013). Table 1 shows the chemical composition of some of the most relevant cereal grains grown worldwide.

In general, cereals are high in their starch content which is high in amylopectin (72-75\%), while amylose accounts for $25-28 \%$. Besides its nutritional importance in cereals, starch plays a functional role when processed and transformed into value-added products due to its gelatinization (in pasta, for example) and retrogradation properties (in cereal-based baked goods) (Rodehutscord et al., 2016).

As for cereals' protein composition, a great variability exists depending on the type of cereal. In general, the amino acid glutamine is the predominant one in most cereals, representing $15-31 \%$ of all amino acids present. Wheat, rye, and barley possess a significant fraction of proline (12-14\%). Leucine (7-14\%) and alanine $(4-11 \%)$ can also be found in high amounts in cereal grains. On the contrary, essential amino acids, such as tryptophan, 
methionine, histidine, and lysine, are present in smaller quantities (0.2-1.0, 1.3-2.9, 1.8-2.2, and 1.4-3.3\%, respectively). However, with recent advances in genetic engineering, there is a possibility of increasing the levels of essential amino acids, as successfully achieved in the case of high-lysine barley and corn (Yu and Tian, 2018).

The germ is the principal storage site for cereal lipids. Among cereals, oat is a significant source of lipid (6-8\%) as opposed to wheat and rye (1.7\%). The fatty acid composition of oat lipids is similar to most cereal grains, with a predominance of linoleic acid (39-69\%), followed by oleic acid (11-36\%) and palmitic acid (18$28 \%$ ). Although triacylglycerols are the primary lipid class in the germ, a small fraction of phospholipids and glycolipids can also be found in the endosperm (Koehler and Wieser, 2013).

Minerals and vitamins are the minor constituents of cereals. In terms of mineral content (calcium, magnesium, potassium, phosphorus, iron, and sodium), cereals are richer than milk, meat, and vegetables but present lower concentrations when compared to pulses. Nevertheless, as staple foods, cereals are consumed in high quantities; thus, being characterized as significant sources of minerals in the diet. The majority of minerals in cereals are concentrated in the bran and the germ. Meanwhile, cereals are rich sources of vitamins from the B-group and tocopherols. Similar to minerals, the vitamins are mainly found in the germ, highlighting the importance of consuming whole grains as opposed to refined cereals (Baniwal et al., 2021).

\section{Phenolic compounds in cereals}

Phenolic compounds are secondary plant metabolites that have the primary role of protecting against oxidative stress, ultraviolet light, and pathogen attack (Shahidi and Naczk, 2003). Structurally, phenolics are characterized by the presence of one or more aromatic rings holding one (monophenols) or more (polyphenols) hydroxyl groups (Duodu, 2011). To date, over 8,000 phenolics have been identified in natural sources, being classified into several subgroups according to their hydroxylation and methylation pattern (Shahidi et al., 2019). Flavonoids, phenolic acids, and tannins (proanthocyanidins and hydrolysable tannins) are the most significant phenolic subclasses; however, lignans and alkylresorcinols are also important phenolic classes in cereals (Cheynier et al., 2013; Shahidi et al., 2019).

Phenolic acids are derived from benzoic and cinnamic acids, being classified into hydroxybenzoic (e.g., $p$-hydroxybenzoic, protocatechuic, vanillic, syringic, and gallic acids) and hydroxycinnamic acids (e.g., $p$-coumaric, caffeic, ferulic, and sinapic acids) (Duodu, 2011; Shahidi et al., 2019). Compounds belonging to this subgroup may also be associated with macromolecules in cereal matrices, such as cellulose, hemicellulose, and pectin, acting as the building blocks supporting the cell wall. This fraction is known as insoluble-bound phenolics and can represent up to $60 \%$ of all phenolic compounds present in selected grains (Shahidi and Yeo, 2016).

Meanwhile, flavonoids are the largest phenolic class in terms of number of compounds. Their basic structural skeleton is C6-C3C6, where two benzene rings are connected to a central heterocyclic ring through a three-carbon bridge. They can be further broken down into flavanones, flavonols, flavan-3-ols, flavones, isoflavones, flavanonols, and anthocyanins, the latter being watersoluble pigments (Shahidi et al., 2019). Cereal grains are abundant sources of flavonoids. In such materials, they can be present either as insoluble-bound or soluble compounds. The soluble flavonoids may occur in the free form, or they can be conjugated with polysaccharides (glycosides) or esterified/etherified to fatty acids (Shahidi and Yeo, 2016).

Monomeric flavonoids can form oligomers and polymers, obtaining a higher degree of complexity. These polyphenols are known as proanthocyanidins; polymeric structures composed of $(+)$-catechin and (-)-epicatechin. Besides flavonoids, phenolic acids can also form polymeric structures known as hydrolysable tannins, which can be either esters of gallic (gallotannins) or ellagic (ellagitannins) acids (Shahidi and Yeo, 2016).

Besides phenolic acids, flavonoids, and tannins, other phenolic groups are also considerable in cereals, namely lignans and alkylresorcinols, the latter being a class of phenolic lipids. Lignans are dimers with a 2,3-dibenzylbutane structure. They are precursors of phytoestrogen, and can occur in cereal grains such as rye, wheat, barley, and oats (Bhattacharya et al., 2010). Meanwhile, alkylresorcinols are amphipathic 1,3-dihydroxybenzene derivatives carrying an alkyl chain (odd-numbered) at position 5 of the benzene ring. They are present in wheat, rye, barley, millet, and maize (Ross et al., 2003a).

The antioxidant power of phenolic compounds is related to most of their known bioactivities. They can function both as primary (free radical scavenging) or secondary (metal chelation) antioxidants. As primary antioxidants, phenolics can donate hydrogen atoms to free radicals, stabilizing them and interrupting the propagation of oxidation. Then, the compound is transformed into a phenoxyl radical, which gains stability by delocalizing its unpaired electron around the aromatic ring. On the other hand, phenolics bearing catechol and galloyl groups can also act as secondary antioxidants by complexing with transition metals, preventing metal-catalyzed initiation of free radicals and formation of reactive oxygen species (Chen et al., 2020).

According to Naczk and Shahidi (2006), flavonoids and phenolic acids in cereals occur as free compounds or conjugated with other molecules, being mainly located in the bran, even though the endosperm and germ also present considerable amounts. Table 2 presents the phenolic composition of major cereal grains.

Zhang et al. (2019) analyzed the pericarp of purple maize and reported a total phenolic content (TPC) of 69.1-229.1 mg of gallic acid equivalents (GAE)/g and total anthocyanin content of 17-93.5 $\mathrm{mg}$ of cyanidin-3-glucoside equivalents $(\mathrm{C} 3 \mathrm{G}) / \mathrm{g}$, varying according to the genotype. The anthocyanins cyanidin-3-glucoside, pelargonidin-2-O-glucoside, peonidin-3-O-glucoside, cyanidin3-(6'-malonylglucoside), pelargonidin-3-(6'-malonylglucoside), and peonidin-3-(6'-malonylglucoside were identified in the samples. Besides, phenolic acids, namely $o$-coumaric, caffeic, vanillic, protocatechuic, and ferulic acids were also identified, as well as flavonoids rutin, luteolin, quercetin, naringenin, and kaempferol. In terms of bioactivities, the authors demonstrated that quercetin, luteolin, and rutin were the main contributors to the anti-inflammatory and anti-diabetic actions displayed by the samples. At the same time, vanillic and protocatechuic acids had a major impact in terms of anti-adipogenic potential.

According to Suriano et al. (2021), among pigmented maize, the purple variety is the richest one in terms of total phenolic $(4,047$ $\mu \mathrm{g}$ GAE/g), total flavonoid $(1,998 \mu \mathrm{g}$ of catechin equivalents (CE)/g), total anthocyanin $(780 \mu \mathrm{g} \mathrm{C} 3 \mathrm{G} / \mathrm{g})$, and total proanthocyanidin $(1,381 \mu \mathrm{g} \mathrm{CE} / \mathrm{g})$ contents, followed by blue maize $(2,416 \mu \mathrm{g}$ $\mathrm{GAE} / \mathrm{g}, 1,739 \mu \mathrm{g} \mathrm{CE} / \mathrm{g}, 216 \mu \mathrm{g} \mathrm{C} 3 \mathrm{G} / \mathrm{g}$, and 1,279 $\mu \mathrm{g} \mathrm{CE} / \mathrm{g}$, respectively) and red maize $(2,373 \mu \mathrm{g}$ GAE/g, 1,664 $\mu \mathrm{g} \mathrm{CE} / \mathrm{g}, 38.9$ $\mu \mathrm{g} \mathrm{C} 3 \mathrm{G} / \mathrm{g}$, and $578 \mu \mathrm{g} \mathrm{CE} / \mathrm{g}$, respectively). Conventional yellow maize had the lowest total phenolics $(1,359 \mu \mathrm{g}$ GAE/g) and flavonoids $(1,162 \mu \mathrm{g} \mathrm{CE} / \mathrm{g})$ and does not contain any anthocyanins.

Pigmented rice is also a remarkable source of anthocyanins. 


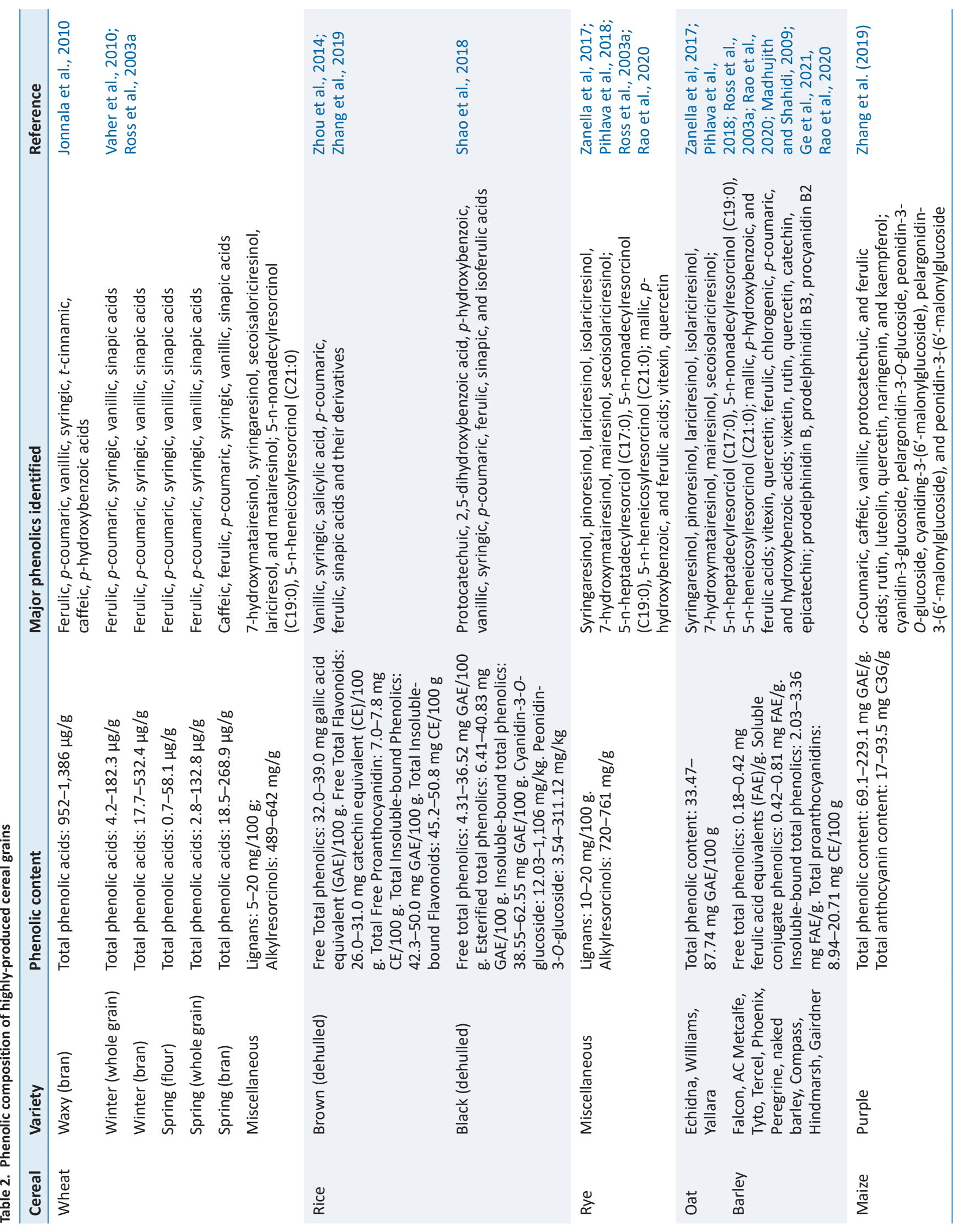




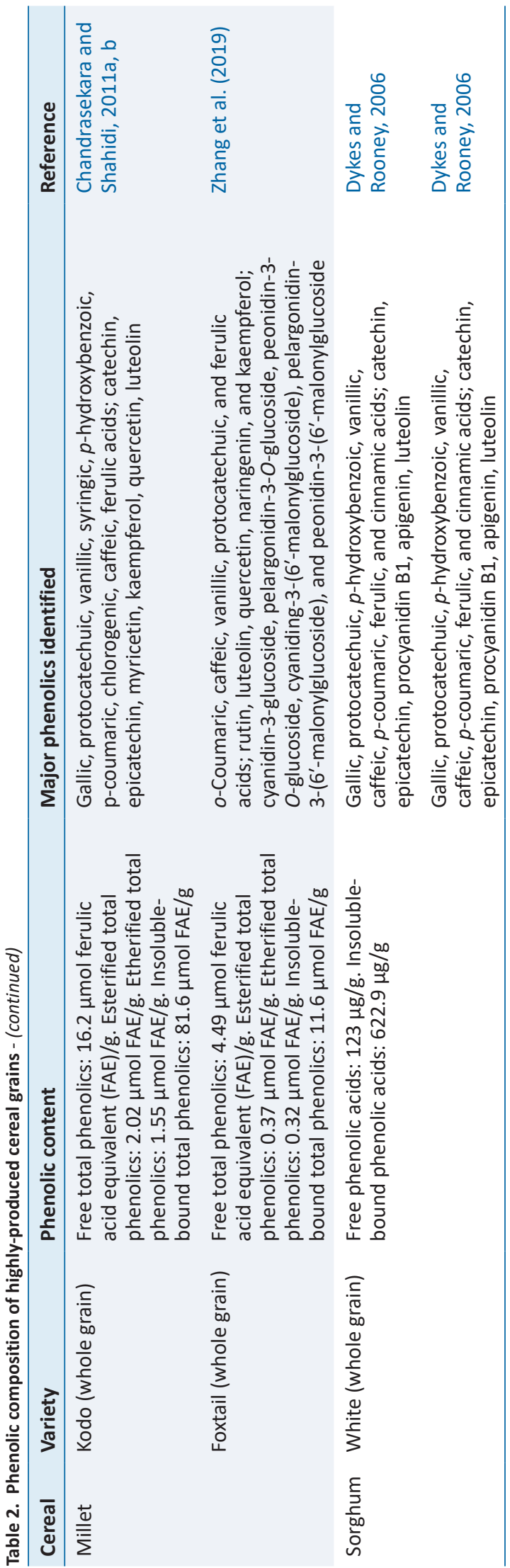

Pang et al. (2018) analyzed the free and insoluble-bound phenolic fractions of whole-grain and bran of white, red, and black rice. It is noteworthy that most samples contained around two times more insoluble-bound than free phenolics. This fraction has also been reported as the most prominent one in maize $(85 \%)$, oats and wheat (75\%) (Adom and Liu, 2002). Liyana-Pathirana and Shahidi (2006) demonstrated that hard and soft whole wheat are mainly composed of insoluble-bound phenolics, as opposed to their free and esterified counterparts. This reinforces the role of cereals as one of the main sources of insoluble-bound phenolics in the diet. This fraction has been associated with improved gut health and reduction of the risk of developing colon cancer (Shahidi and Yeo, 2016). According to Pang et al. (2018), rice bran has a phenolic content of almost four times higher than the whole grain, confirming that these compounds are preferably concentrated in the outer layers of cereal grains. Pigmented rice (red and black) is superior to white rice in terms of phenolic content since it has a significant amount of anthocyanins, which is lacking in white rice. Ferulic and $p$-coumaric acids were among the main phenolic acids identified in both white and pigmented rice. Isoferulic acid was also found in high amounts in white rice, whereas pigmented rice varieties showed a significant content of vanillic acid.

Proanthocyanidins are also relevant in pigmented rice. Shao et al. (2018) investigated the proanthocyanidin composition of brown, red, and black rice genotypes. Among all samples, proanthocyanidins were only detected in red rice, ranging from 58.13 to 254.8 $\mathrm{mg} \mathrm{CE} / 100 \mathrm{~g}$, depending on the genotype. According to the authors, the majority of identified proanthocyanidins were oligomers of 5-8 units (40\%), while polymers (over ten units) accounted for $29 \%$.

Proanthocyanidins are also present in barley, mainly in the form of oligomeric prodelphinidins and procyanidins. These compounds are involved in the formation of haze in beer. Besides proanthocyanidins, barley presents a rich and diverse phenolic profile, including phenolic acids, their esters and glycosides, anthocyanins, and lignans. The free phenolic fraction of barley is primarily composed of ferulic acid (especially the bran). On the other hand, barley`s insoluble-bound phenolic fraction is abundant in $p$-hydroxybenzoic acid.

Millet grains play an important role in the diet of several Asian and African populations, besides being used as an ingredient in multigrain and gluten-free cereal products. This cereal has a rich and diverse phenolic composition, especially in regard to its insoluble-bound fraction. According to Chandrasekara and Shahidi (2011a, b), over 50 phenolic compounds were identified among various whole millet grain varieties. Most phenolic compounds were detected in the insoluble-bound fraction, which accounted for an average TPC of $17.3 \mu \mathrm{mol}$ ferulic acid equivalent (FAE)/g, followed by free $(6.89 \mu \mathrm{mol} \mathrm{FAE} / \mathrm{g})$ etherified $(2.05 \mu \mathrm{mol} \mathrm{FAE} / \mathrm{g})$, and esterified $(0.99 \mu \mathrm{mol} \mathrm{FAE} / \mathrm{g})$ phenolics. Among the phenolic groups detected, phenolic acids and their derivatives (dehydrodiferulates and dehydrotriferulates), flavan-3-ols monomers and dimers, flavonols, flavones, flavanonols, and proanthocyanidins were most abundant. The insoluble-bound fraction was mainly composed of hydroxycinnamic acids and their derivatives (e.g., chlorogenic, caffeic, $p$-coumaric, ferulic acids). Meanwhile, flavonoids (e.g., catechin, epicatechin, gallocatechin, epigallocatechin, procyanidins B1 and B2) accounted for most of the free phenolics.

Besides its rich phenolic composition, millet has also been shown to present numerous bioactivities. Millet phenolics have demonstrated protection against copper-induced human lowdensity lipoprotein (LDL) oxidation (Chandrasekara and Shahidi, 2012). The accumulation of oxidized LDL can lead to the formation of atheromatous plaques that can eventually cause atherosclerosis and associated cardiovascular diseases. Phenolics 
from Kodo millet were able to suppress in $40 \%$ the formation of conjugated dienes, a product of LDL oxidation. In another study (Chandrasekara and Shahidi, 2011a), millet's soluble (from whole grain, dehulled grain, and hulls) and insoluble-bound (from whole grain) phenolics exerted protection against free radical-induced supercoiled DNA strand scission in a dose-dependent manner. At a concentration of $0.5 \mathrm{mg} / \mathrm{mL}$, phenolic extracts displayed oxidation inhibition varying from 46 to $96 \%$ in the presence of hydroxyl radicals and from 31 to $95 \%$ in the presence of peroxyl radicals. The attack on the DNA molecule by free radicals can lead to base modification and strand scission, inducing mutagenesis, which could further progress to cancer development. Millet phenolics have been demonstrated to be able to suppress this effect. Besides, phenolics from whole millet grains have also shown inhibition of human colon adenocarcinoma cell proliferation with efficiency ranging from 28 to $100 \%$, which lends support to the anticancer potential of millet phenolics (Chandrasekara and Shahidi, 2011b).

Cereals are the major source of lignan in the Western diet, with wheat and rye having the highest contents of lignan among all cereals, especially their bran fraction. In wheat, for example, $70-85 \%$ of the grain lignan is located in the bran, which accounts for 5-20 $\mathrm{mg} / 100 \mathrm{~g}$ on average, depending on the variety. Rye bran possesses a similar content, ranging from 10 to $20 \mathrm{mg} / 100 \mathrm{~g}$. The most commonly found lignans in wheat are 7-hydroxymatairesinol, syringaresinol, secoisaloriciresinol, lariciresinol, and matairesinol. Meanwhile, in rye, the major lignans encountered are syringaresinol, pinoresinol, lariciresinol, isolariciresinol, 7-hydroxymatairesinol, mairesinol, secoisolariciresinol (Zanella et al., 2017; Pihlava et al., 2018). Besides rye and wheat, other cereals present smaller amounts of lignan, namely barley (whole grain flour), buckwheat (whole grain flour), maize (whole grain), and oat (whole grain flour) at average concentrations of $0.28,0.76,0.33$, and $0.65 \mathrm{mg} / 100 \mathrm{~g}$, respectively (Rodríguez-García et al., 2019).

Besides their well-documented antioxidant properties, some lignans can also act as regulators of the activity of specific nuclear receptors (e.g., estrogen receptors, peroxisome proliferator activate receptor gamma, liver $\mathrm{X}$ receptor alpha) that are involved in glucose and fatty acid metabolism, as well as insulin action. This suggests that they could efficiently be used for selected physiological functions (Zanella et al., 2017).

In the food matrix, lignans occur as esters, glycosides, and polymers. Upon consumption, lignan glycosides undergo deglycosylation, being further converted to glucuronidated, methylated, and sulfated derivatives in the liver. These metabolites can be detected in the urine. However, the non-metabolized fraction of glycosylated lignans is directed to the colon, where they are converted into enterolignans, enterolactone, and enterodiol by the gut microbiota. Evidence suggests that the presence of such metabolites in the gut stimulates the colonization of probiotic microorganisms, decreasing the risk of colorectal cancer. However, the exact mechanism for this effect has yet to be clarified (Zanella et al., 2017; Fu et al., 2020).

Among cereal grains, alkylresorcinols (AR) are abundantly present in wheat $(489-642 \mathrm{mg} / \mathrm{g})$ and rye $(720-761 \mathrm{mg} / \mathrm{g})$. To a lesser extent, they can also be found in barley, millet, and maize (Ross et al., 2003a). In this raw material, common AR homologues include 5-n-pentadecylresorcinol (C15:0), 5-n-heptadecylresorciol (C17:0), 5-n-nonadecylresorcinol (C19:0), 5-n-heneicosylresorcinol (C21:0), 5-n-tricosylresorcinol (C23:0), and 5-n-pentacosylresorcinol (C25:0). Rye is predominantly composed of $\mathrm{C} 17: 0$ (23-25\%), C19:0 (31-32\%), and C21:0 (22-25\%) homologues, whereas C19:0 (29-35\%) and C21:0 (46-51\%) represent the major AR contribution to wheat (Ross et al., 2003a). According to Landberg et al. (2008), ARs are mainly located in the intermediate layers (inner pericarp, hyaline layer, and testa) of the wheat kernel $(16.2-16.4 \mathrm{mg} / \mathrm{g})$, followed by the peripheral layers (3.9-4.0 $\mathrm{mg} / \mathrm{g})$.

Similar to other phenolic compounds, ARs have also been reported to possess antioxidant and antimicrobial activities. Besides, observational and in vitro evidence suggests the anticarcinogenic potential of this group. Reports have pointed out a risk reduction of around $52-66 \%$ of distal colon cancer when nanomolar concentrations of ARs are present in the plasma. In addition, ARs have demonstrated high cytotoxicity toward cancerous cell lines (Kruk et al., 2017; Fu et al., 2018).

ARs have been indicated as possible biomarkers to correlate whole-grain cereals consumption with health benefits due to their high absorption rate in humans. Ross et al. (2003b) administered a high-AR diet based on the consumption of rye bran-enriched bread to ten ileostomy-operated subjects and verified that around $40 \%$ of the AR present in the diet were recovered in effluent from the small intestine, while $60 \%$ had been absorbed in the small intestine and further metabolized. The authors also suggested that mediumchain AR homologues (C17:0, C19:0) are taken up at a higher frequency than their long-chain counterparts (C23:0, C25:0).

\section{Effect of processing on cereal phenolics}

After harvest and before reaching the consumer's table, cereals undergo a series of post-harvest processes that can vary according to the type of grain and specific destination. Firstly, the harvested grains are prepared for storage, being subsequently subjected to cleaning, husk removal, and size reduction (in some cases) in the stage known as primary processing. Then, cereals can be commercialized as such, or they can be transformed into value-added products. This stage is known as secondary processing and is characterized by the use of primary products (whole grains, flakes, or flour) as ingredients for breakfast cereals, snacks, cereal bars, doughs, batters, and noodles, among others. In secondary processing, various unit operations (e.g., puffing, flaking, fermentation, baking, extrusion) can be used alone or in combination (Food and Agriculture Organization, 2021).

Due to the drastic transformation that cereal grains undergo during this step, secondary processing is generally the focus of studies interested in the effect of processing on cereals' bioactive compounds. However, evidence points out that even primary processing and post-harvest handling of cereals may affect their bioactive content and composition. Table 3 summarizes the possible effects of several cereal processing techniques on their phenolic composition and bioactivities.

\subsection{Post-harvest treatment and storage}

Cereal harvesting usually begins before the grain is fully ripe and continues until mold and insect damage becomes extensive. The harvest is subsequently subjected to threshing, which is characterized by removing the grains from the rest of the plant. Winnowing follows next, separating the grains from the chaff or straw. A crucial stage in cereal post-harvest treatment is the drying stage, responsible for reducing moisture content to $10-15 \%$. This can happen through sun-drying, a cost-effective technique, or artificial drying in humid locations. Finally, the dried grains are ready to be stored in bulk until further processing. During storage, they should be constantly inspected for spoilage and moisture content. Re-drying may be necessary in some cases (Schmidt et al., 2018). 
Table 3. Effect of common cereal processing techniques on their phenolic composition

\begin{tabular}{|c|c|c|c|}
\hline Cereal & Process & Effect & Reference \\
\hline Barley & $\begin{array}{l}\text { Infrared and hot } \\
\text { air drying }\left(60^{\circ} \mathrm{C}\right)\end{array}$ & $\begin{array}{l}\text { Infrared drying released bound phenolics, increasing the phenolic } \\
\text { content in regards to the hot air drying and original samples }\end{array}$ & Ge et al., 2021 \\
\hline Millet & Dehulling & $\begin{array}{l}\text { Reduction of total phenolic content. Reduction of oxygen radical } \\
\text { absorbance capacity and hydroxyl-radical scavenging capacity. } \\
\text { Reduction of the concentration of } p \text {-coumaric and ferulic acids }\end{array}$ & $\begin{array}{l}\text { Chandrasekara and } \\
\text { Shahidi, 2011a, b }\end{array}$ \\
\hline Rice & Parboiling & $\begin{array}{l}\text { Release of bound phenolics, increasing the contents of } p \text {-coumaric, } \\
\text { syringic, protocatechuic, and ferulic acids compared to raw sample }\end{array}$ & $\begin{array}{l}\text { Thammapat } \\
\text { et al., } 2016\end{array}$ \\
\hline Wheat & $\begin{array}{l}\text { Steam explosion } \\
\left(215^{\circ} \mathrm{C} \text { for } 120 \mathrm{~s}\right)\end{array}$ & Increased concentration of soluble phenolic acids & Liu et al., 2016 \\
\hline $\begin{array}{l}\text { Brown rice, } \\
\text { wheat, and oat }\end{array}$ & Extrusion cooking & $\begin{array}{l}\text { Enhanced content of insoluble-bound phenolics. } \\
\text { Increased bioaccessibility of wheat phenolics }\end{array}$ & Zeng et al., 2016 \\
\hline Maize & Roasting (17 min) & $\begin{array}{l}\text { Decreased total phenolic and flavonoid contents. Diminished } \\
\text { free radical scavenging activity and iron chelating ability }\end{array}$ & Oboh et al., 2010 \\
\hline
\end{tabular}

\subsubsection{Cultivation and harvesting}

Variations regarding the phenolic profile of cereals may take place even before harvesting begins. Climate conditions, agricultural practices, and soil quality play a role in the bioactive composition of such feedstock. This becomes particularly evident when studying the phenolic composition of a specific cereal grown in different locations. Emmons and Peterson (2001) reported that oats cultivated at several locations in Wisconsin presented significant differences in their phenolic content and profile. Horvat et al. (2020) analyzed the phenolic acid content of wheat, corn, and barley over two crop years. Insoluble-bound phenolic acids ( $p$-hydroxybenzoic, vanillic, caffeic, ferulic, syringic, $p$-coumaric, $o$-coumaric acids) showed higher contents in the crop year where precipitation and temperature conditions were more extreme, due to a response to abiotic stress toward drastic environmental conditions. A similar outcome was observed for barley and corn, which biosynthesized more insoluble-bound phenolic acids during intense precipitation periods, a response to high water stress.

Additionally, the agronomic management of crops can also affect phenolics. According to Rempelos et al. (2018), using mineral NPK fertilizers causes the reduction of phenolic acid and flavonoid contents in wheat. Meanwhile, herbicides, fungicides, and growth regulators do not cause significant variations in the same parameters. On the other hand, Stumpf et al. (2015) observed that enriching the soil with nitrogen supply in wheat crops positively affects the concentration of free phenolics while reducing the amount of conjugated soluble phenolics and maintaining insoluble-bound phenolics within the same range.

Cultivation system has been found to influence phenolic yield. Fares et al. (2019) cultivated wheat genotypes under organic and traditional systems. Total phenolic content was $10 \%$ higher in the wheat grown under the organic system. However, when analyzing the individual contents of free vanillin and ferulic acid, as well as insoluble-bound vanillic, caffeic, syringic, $p$-coumaric, and ferulic acids, as well as vanillin, the authors did not find significant differences between the organic and the conventional systems.

\subsubsection{Drying}

Setyaningsih et al. (2016) investigated how sun-drying affects phenolics in rice during the post-harvest stage. No major differences were observed in the content of protocatechuic, vanillic, protocatechuicaldehyde, $p$-hydroxybenzoic, $p$-hydroxybenzaldehyde, ferulic, sinapic, syringic, and $p$-coumaric acids, and vanillin between the wet and the dried paddy. Ge et al. (2021) studied unconventional drying techniques (infrared and hot air drying at 60 ${ }^{\circ} \mathrm{C}$ ) on the post-harvest processing of naked barley. Samples were dried to $10 \%$ moisture. Infrared drying was found to preserve the phenolic compounds better, even leading to higher yields when compared to the original sample. This effect was due to the fact that kinetic energy is converted to thermal energy during infrared drying, releasing bound compounds. Although hot air drying also presented higher phenolic yields than the original sample, infrared drying was deemed more efficient in releasing bound phenolics while avoiding losses.

Wanyo et al. (2014) studied the impact of hot air drying (120 ${ }^{\circ} \mathrm{C} / 30 \mathrm{~min}$ ), far-infrared (FIR) radiation, and cellulase treatment on the phenolic content and antioxidant activity of rice bran and husk. Generally, cellulase-aided process and hot-air drying were able to preserve phenolics and their antioxidant activity, measured by 2,2-diphenyl-1-picrylhydrazyl (DPPH) and ferric reducing ability of plasma (FRAP) assays, with little or no significant difference in such parameters in regard to the raw materials. However, FIR managed to increase total phenolic and flavonoid contents as well as antioxidant capacity, which is similar to the outcome observed by Ge et al. (2021). Some interesting observations include the effect of cellulase, which significantly increased the content of vanillic acid while drastically reducing ferulic acid concentration.

\subsubsection{Storage}

Once post-harvest treatment has taken place, cereals can be stored. The storage period and conditions are also crucial for the overall quality of the grains and, especially, their bioactive compounds. Extreme $\mathrm{pH}$, time, temperature, and UV exposure conditions should be avoided, as they may degrade the bioactive content and antioxidant power. Sripum et al. (2016) stored Thai Jasmine parboiled germinated brown rice for six months under 30 and $40{ }^{\circ} \mathrm{C}$. Neither temperature affected the total phenolic content and antioxidant activity (measured by DPPH, oxygen free radical scavenging activity, and FRAP), as such parameters kept stable and without major variances during the six-month storage. The rice samples were maintained vacuum-sealed for the whole period, which could 
have contributed to this outcome. The lack of oxygen avoided the formation of oxidation products, leading to the preservation of phenolics and their antioxidant capacity.

De Oliveira et al. (2017) evaluated three storage temperatures $\left(4,25\right.$, and $\left.40{ }^{\circ} \mathrm{C}\right)$ for sorghum grain and flour; they did not detect changes in the contents of 3-deoxyanthocyanins (luteolinidin, 5-methoxyluteolinidin, apigeninidin, and 7-methoxyapigeninidin) and total proanthocyanidins. On the other hand, storage time considerably affected sorghum's phenolics. Up to 60 days of storage, most phenolics in sorghum showed a reduction in their content, stabilizing after this period. After 180 days, $86.7-86.8 \%$ of all phenolics were retained. The proanthocyanidins were the most affected group, retaining only about $56.6-85.3 \%$ after the same period.

\subsection{Primary processing}

Prior to advanced processing, cereals grains are cleaned and graded based on their size. Depending on the type of cereal, dehulling, which consists of the removal of unpalatable hulls or shells, can be necessary. If grains are used as primary material for flour production, a milling step will be conducted. This can generally happen in a plate, hammer, or roller mill, according to grain type and production scale. A common process for rice, parboiling is an optional step, and it involves pre-cooking the grain by soaking and heating it, loosening the hulls, and aiding in grain preservation and cooking efficiency. In addition, for white rice production, a polishing stage is required. Although popular in some cultures, nutritionally speaking, white rice is less appealing than its brown counterpart due to the removal of the bran and the germ. These fractions concentrate the highest proportion of bioactive compounds, including phenolics, while the endosperm is mainly constituted by carbohydrates (Schmidt et al., 2018; Food and Agriculture Organization, 2021).

\subsubsection{Dehulling and parboiling}

Dehulling is the process of removing the outer layers of grains, and this is usually carried out as a part of the milling process (Duodu, 2011). This technique reduces the content of phenolic compounds, negatively impacting the antioxidant activity of processed grains (Duodu, 2011). Živković et al. (2021) conducted cold dehulling on germinated buckwheat seeds and analyzed its effect on phenolic profile and antioxidant activity. Regardless of germination time, the dehulled buckwheat showed a sharp decrease in all identified compounds' content compared to the hulled sample. Some insoluble-bound phenolics quantified in the hulled samples could not be detected in their dehulled counterparts, namely orientin, rutin, and hyperin. As expected, antioxidant capacity measured by DPPH declined considerably once the seeds were dehulled. The same trend was observed by Dlamini et al. (2007) in sorghum.

Chandrasekara and Shahidi (2011a, b) detected that Pearl millet significantly reduced its total phenolic content, negatively affecting the phenolics' bioactivities (e.g., oxygen radical absorbance capacity (ORAC) and hydroxyl-radical scavenging capacity). Besides, two of the major phenolic acids present in millet, ferulic and $p$-coumaric acid, had their concentrations diminished by 39 and $52 \%$, respectively. A similar study by Madhujith et al. (2006) was carried out in pearled barley fractions. The outermost fractions concentrated most phenolic compounds (TPC of 4.16-6.26 $\mathrm{mg} \mathrm{FAE} / \mathrm{g}$ ), dropping considerably as the innermost fractions were reached (TPC of $0.17-0.51 \mathrm{mg} \mathrm{FAE} / \mathrm{g}$ ). Additionally, free caffeic and $p$-coumaric acids were only detected in the outermost fractions of the grains.

Alkylresorcinols are also affected by the dehulling process. According to Giambanelli et al. (2018), whole wheat kernels showed a higher alkylresorcinol content than their hulled counterpart. After the pearling process, the authors analyzed the waste fractions in order to determine where the phenolic lipids were mostly concentrated and found that the bran was the main location of akylresorcinols. This fraction was also the one showing the highest antioxidant capacity. Wheat bran was determined to be rich in unsaturated alkylresorcinols homologues, mainly C18:1 and C18:2.

In contrast, parboiling has been reported to improve phenolic concentration considerably. Thammapat et al. (2016) compared two parboiling methods for glutinous rice. The traditional method consisted of soaking the rice in water for $24 \mathrm{~h}$ at room temperature, followed by steaming at $100{ }^{\circ} \mathrm{C}$ for $1 \mathrm{~h}$ and sun-drying for $12-14 \mathrm{~h}$. In the alternative method, soaking was conducted for only $6 \mathrm{~h}$ at 60 ${ }^{\circ} \mathrm{C}$, instead of room temperature. Also, the steaming temperature was $120^{\circ} \mathrm{C}$ for $15 \mathrm{~min}$, followed by cabinet-drying at $50{ }^{\circ} \mathrm{C}$ for 12-14 h. Both parboiled rice samples exhibited higher content of phenolic acids (traditional $-52.24 \mu \mathrm{g} / \mathrm{g}$, alternative $-65.39 \mu \mathrm{g} / \mathrm{g}$ ) than raw rice $(45.94 \mu \mathrm{g} / \mathrm{g})$, with $p$-coumaric, syringic, protocatechuic, and ferulic acids as the compounds showing the sharpest increase. According to the authors, a possible explanation for this effect could be the release of bound phenolics due to the thermal treatment. Similar outcomes have been observed for red rice $\mathrm{Hu}$ et al., 2017) and millet (Bora et al., 2019).

\subsection{Secondary processing}

Secondary processing of cereals involves operations responsible for further transforming the grains into more palatable and digestible forms or using them as ingredients for added-value products. A vast array of techniques can be employed at this stage, such as extrusion, fermentation, high-pressure processing, steam explosion, and roasting.

\subsubsection{Steam explosion and high-pressure processing}

Steam explosion can be used for the pre-treatment of cereals in order to release bound phenolics (Baruah et al., 2018; Gong et al., 2012). During this treatment, phenolic compounds are exposed to high-pressure steam $(0.69-4.83 \mathrm{MPa})$ and high temperature ranging from 160 to $260{ }^{\circ} \mathrm{C}$ for only a few seconds/minutes (Baruah et al., 2018; Rabemanolontsoa and Saka, 2016; Gong et al., 2012). After subjecting the cereal matrix to high pressure, a sudden decline in pressure results in the explosion and releases water, thus leading to the disruption of the cell wall structure (Baruah et al., 2018; Singh et al., 2015). Throughout the process, acetic acid is liberated from xylan hemicelluloses, which catalyzes further reactions, such as the hydrolysis of hemicellulose. Therefore, this treatment is called autohydrolysis (Baruah et al., 2018; Rabemanolontsoa and Saka, 2016). The advantages of steam explosion include low environmental impact and low cost as it needs limited chemicals in a simplified process (Baruah et al., 2018; Pielhop et al., 2016).

The efficiency of steam explosion can be affected by steam temperature, residence time, sample size, and moisture content (Baruah et al., 2018; Rabemanolontsoa and Saka, 2016). According to Rabemanolontsoa and Saka (2016), lower temperatures and longer residence times are more favorable for extracting bound phenolics. Under drastic temperature conditions, solubilized carbohydrates such as hemicellulose undergo further secondary reactions to form carboxylic acids and soluble polymers (Gong et al., 2012; Sun et 
al., 2005). In addition, reactive precursors cause condensation with lignin, resulting in a lower phenolic yield (Sun et al., 2005). Gong et al. (2012) studied the effect of steam explosion at $220^{\circ} \mathrm{C}$ for $10-$ $120 \mathrm{~s}$ on phenolic compounds in Tibetan barley bran (dehulled). This study has shown that the steam explosion pre-treatment effectively decomposes carbohydrates in this cereal, which results in a high yield of total free phenolic acids. Total antioxidant capacity was also increased at $220^{\circ} \mathrm{C}$ for $120 \mathrm{~s}$ (Gong et al., 2012).

Similarly, the amount of soluble phenolic acids in wheat bran and their antioxidant properties were significantly increased after steam explosion at $215{ }^{\circ} \mathrm{C}$ for $120 \mathrm{~s}$ (Liu et al., 2016). A recent study has shown that steam pressure may also be an important parameter, and milder conditions are more effective for increasing the yield of phenolics, contributing to improved bioactivities. Li et al. (2020) reported that steam explosion enhanced the anti-proliferative activity of phenolics in Tartary buckwheat bran on human colon and liver cancer cells. These results highlight the potential of steam explosion as an effective pre-treatment to promote enhanced bioaccessibility of bound phenolics from cereals and other plant foods.

Other technologies have also been investigated for the release of bound phenolics from cereals. Luo et al. (2020) used high-pressure boiling on sorghum hull. This method was able to increase the content of free procyanidins and total phenolics by $35.92-58.87$ and $6.20-18.70 \%$, respectively, compared to traditional hot water processing. The disruption of bound phenolics caused by the combination of high temperature and pressure, along with the inactivation of endogenous polyphenol oxidase, prevented the oxidation and degradation of phenolics.

\subsubsection{Extrusion cooking}

Extrusion cooking is largely used in the food industry to manufacture pasta, noodles, and cereal-based snacks. This continuous process uses a combination of high temperature, high pressure, and high shearing to plasticize the raw material so it can be conveyed and forced to flow through a die or specific shape. In terms of phenolics, heat-sensitive compounds may be susceptible to degradation and polymerization, decreasing their concentration in the final product. On the other hand, extrusion also causes the disruption of cell walls, breaking the covalent bonds between bound phenolics and macromolecules, which results in an increased bioaccessibility of this phenolic fraction (Wang et al., 2014). Zeng et al. (2016) studied the effect of extrusion on brown rice, wheat, and oat phenolics and their bioaccessibility. All cereal grains had their insoluble-bound phenolic content significantly increased after the extrusion cooking. The bioaccessibility varied according to the grain type. While $11.60 \%$ of phenolics from raw wheat were bioaccessible, extrusion marginally improved it to $12.48 \%$. On the other hand, the bioaccessibility of oat phenolics dropped from $29.83 \%$ (raw) to $21.78 \%$ (extruded). Brown rice kept the same bioaccessibility levels before and after extrusion (around 20\%). According to the authors, phenolic bioaccessibility was mainly dependent on the cereal type and the way the compounds occur in the matrix since higher phenolic content did not translate into higher bioaccessibility.

\subsubsection{Fermentation, roasting, and popping}

Biological treatments, such as fermentation, can also be used to enhance the bioefficiency of cereal phenolics. During this process, enzymes are produced and released, promoting the hydrolysis of phenolic glycosides, liberating them as free compounds (Wang et al., 2014). Bvochora et al. (2005) reported an increase in the total phenolic content of sorghum during alcoholic fermentation; this brought about higher concentrations of $p$-hydroxybenzoic acid and $p$-hydroxybenzyl alcohol. Meanwhile, the levels of $p$-hydroxybenzaldehyde decreased.

Total phenolic content of buckwheat, barley, wheat, and rye has been shown to increase after fermentation with Lactobacillus rhamnosus (L. rhamnosu) and Saccharomyces cerevisiae (S. cerevisiae) (Đorđević et al., 2010). A recent study on millet has also shown that fermentation with Lactobacilli increased the total phenolic content due to the release of bound polyphenols. The fermentation treatment also enhanced the in vitro effect of millet phenolics on type 2 diabetes markers (Balli et al., 2020).

Roasting is also commonly used in the processing of several cereals, including maize and sorghum. Oboh et al. (2010) roasted yellow and white maize for 17 min and observed a significant decrease in their total phenolic and flavonoid contents, which was reflected in the lower free radical scavenging and iron chelating ability of the samples compared to the raw cereal. Irondi et al. (2019) obtained similar results when studying the roasting of red sorghum at 150 and $180^{\circ} \mathrm{C}$ for $20 \mathrm{~min}$. Besides reducing the levels of phenolic compounds, enzyme inhibitory activities (pancreatic lipase, alpha-amylase, alpha-glucosidase, xanthine oxidase, and angiotensin 1-converting enzyme) were slightly reduced in comparison to the original sample, with the roasting at $180^{\circ} \mathrm{C}$ displaying the most negative effect. These outcomes can be explained by the fact that the high temperature during the roasting process can lead to thermal decomposition and heat-induced oxidation of phenolics.

Popping is used to expand cereals and promote starch gelatinization. In this process, grains are subjected to a high-temperature treatment for a short time, which creates superheated vapor inside the grains. The internal pressure causes the endosperm to expand, breaking the outer layer. This process imparts desirable sensory characteristics to cereal-based snacks, and it is commonly used to produce popcorn, popped rice, and popped sorghum, among others (Mishra et al., 2014). Bagchi et al. (2021) analyzed how different processing techniques, including popping, affect phenolic acids, flavonoids, and anthocyanins in black rice. Hydroxybenzoic acids were the predominant phenolic acid class in rice following the popping process, with $75.78 \mu \mathrm{g} / \mathrm{g}$. Gallic acid was the major phenolic acid detected in popped rice, followed by protocatechuic and vanillic acids. Moreover, $83 \%$ of all polyphenols quantified in the product were in the free form. In terms of flavonoids, kaempferol and catechin showed high concentrations in popped rice, with 9.19 $\mu \mathrm{g} / \mathrm{g}$ and $8.38 \mu \mathrm{g} / \mathrm{g}$, respectively. Anthocyanins were the main phenolic group found in popped rice, with an average of $119.4 \mu \mathrm{g} / \mathrm{g}$, higher than the content found in beaten and milled rice but lower than the anthocyanin levels in boiled and puffed rice. Among all processes, popping produced the rice product with the highest antioxidant activity, measured by DPPH and FRAP assays. This outcome was due to the fact that the thermal treatment in popping $\left(177{ }^{\circ} \mathrm{C}\right.$ for $\left.40-50 \mathrm{~s}\right)$ was not drastic enough to degrade the phenolic compounds.

\section{Conclusions and final remarks}

The economic and nutritional importance of cereal grains is undeniable. They provide a great portion of the carbohydrate, protein, lipid, vitamins, and minerals consumed in the diet of many populations, helping to fulfill the global food demand. Nevertheless, the consumption of cereals is also advantageous from a bioactive 
standpoint, with phenolic compounds assuming the role of providing benefits due to their antioxidant power and related bioactivities.

Notwithstanding, cereals undergo post-harvest treatments, primary, and in some cases, secondary processing before reaching the consumer's table. Thus, different processing techniques show distinct effects on cereal phenolics. Therefore, such factors should be analyzed in a case-by-case manner. Processes that remove the outer layers of cereal grains, such as polishing of rice and dehulling of wheat and other cereals, significantly reduce the phenolic content due to the exclusion of fractions that are highly concentrated in these compounds. On the other hand, mild drying techniques and technologies involving the use of high-pressure can enhance the phenolic composition of cereals since they promote the release of the insoluble-bound phenolic fraction, increasing bioaccessibility parameters. Finally, drastic processing, such as extrusion cooking and roasting, may negatively affect cereal phenolics, considerably reducing their biological activities, but may enhance consumer acceptability due to generation of desirable aroma compounds as well as Maillard reaction products that may possess their own activities.

Therefore, cereal consumption, especially in their whole-grain form, should be stimulated given their large variety of polyphenols, including phenolic acids, flavonoids, tannins, and anthocyanins (pigmented cereals) that are associated with reduced risk of developing chronic diseases, such as colorectal cancer, cardiovascular ailments, and type 2 diabetes. In addition, processing conditions must be carefully analyzed in order to increase the bioefficiency of cereal phenolics and avoid their degradation.

\section{Acknowledgments}

We thank the Natural Science and Engineering Research Council (NSERC) of Canada for support in the form of a Discovery Grant to FS.

\section{References}

Adom, K.K., and Liu, R.H. (2002). Antioxidant activity of grains. J. Agric. Food Chem. 50(21): 6182-6187.

Bagchi, T.B., Chattopadhyay, K., Sivashankari, M., Roy, S., Kumar, A., Biswas, T., and Pal, S. (2021). Effect of different processing technologies on phenolic acids, flavonoids and other antioxidants content in pigmented rice. J. Cereal Sci. 100: 103263.

Balli, D., Bellumori, M., Pucci, L., Gabriele, M., Longo, V., Paoli, P., Melani, F., Mulinacci, N., and Innocenti, M. (2020). Does fermentation really increase the phenolic content in cereals? A study on millet. Foods 9(3): 303.

Baniwal, P., Mehra, R., Kumar, N., Sharma, S., and Kumar, S. (2021). Cereals: Functional constituents and its health benefits. Pharma. Innov. 10(3): 01-07.

Baruah, J., Nath, B.K., Sharma, R., Kumar, S., Deka, R.C., Baruah, D.C., and Kalita, E. (2018). Recent Trends in the Pretreatment of Lignocellulosic Biomass for Value-Added Products. Front. Energy Res. 6: 141.

Bhattacharya, A., Sood, P., and Citovsky, V. (2010). The roles of plant phenolics in defence and communication during Agrobacterium and Rhizobium infection. Mol. Plant Pathol. 11(5): 705-719.

Bora, P., Ragaee, S., and Marcone, M. (2019). Effect of parboiling on decortication yield of millet grains and phenolic acids and in vitro digestibility of selected millet products. Food Chem. 274: 718-725.

Bvochora, J.M., Danner, H., Miyafuji, H., Braun, R., and Zvauya, R. (2005). Variation of sorghum phenolic compounds during the preparation of opaque beer. Process Biochem. 40(3-4): 1207-1213.

Chandrasekara, A., and Shahidi, F. (2011a). Determination of antioxidant activity in free and hydrolyzed fractions of millet grains and characterization of their phenolic profiles by HPLC-DAD-ESI-MSn. J. Funct. Foods 3(3): 144-158.

Chandrasekara, A., and Shahidi, F. (2011b). Antiproliferative potential and DNA scission inhibitory activity of phenolics from whole millet grains. J. Funct. Foods 3(3): 159-170.

Chandrasekara, A., and Shahidi, F. (2012). Antioxidant phenolics of millet control lipid peroxidation in human LDL cholesterol and food systems. J. Am. Oil Chem. Soc. 89(2): 275-285.

Chawda, P.J., Shi, J., Xue, S., and Young Quek, S. (2017). Co-encapsulation of bioactives for food applications. FQS 1(4): 302-309.

Chen, J., Yang, J., Ma, L., Li, J., Shahzad, N., and Kim, C.K. (2020). Structureantioxidant activity relationship of methoxy, phenolic hydroxyl, and carboxylic acid groups of phenolic acids. Sci. Rep. 10(1): 1-9.

Cheynier, V., Comte, G., Davies, K.M., Lattanzio, V., and Martens, S. (2013). Plant phenolics: Recent advances on their biosynthesis, genetics, and ecophysiology. Plant Physiol. Biochem. 72: 1-20.

De Oliveira, K.G., Queiroz, V.A.V., de Almeida Carlos, L., de Morais Cardoso, L., Pinheiro-Sant'Ana, H.M., Anunciação, P.C., Menezes, C.B., da Silva, E.C., and Barros, F. (2017). Effect of the storage time and temperature on phenolic compounds of sorghum grain and flour. Food Chem. 216: 390-398.

Dlamini, N.R., Taylor, J.R., and Rooney, L.W. (2007). The effect of sorghum type and processing on the antioxidant properties of African sorghum-based foods. Food Chem. 105(4): 1412-1419.

Đorđević, T.M., Šiler-Marinković, S.S., and Dimitrijević-Branković, S.I. (2010). Effect of fermentation on antioxidant properties of some cereals and pseudo cereals. Food Chem. 119(3): 957-963.

Duodu, K.G. (2011). Effects of processing on antioxidant phenolics of cereal and legume grains. Advances in Cereal Science: Implications to Food Processing and Health Promotion. American Chemical Society, pp. 31-54.

Dykes, L., and Rooney, L.W. (2006). Sorghum and millet phenols and antioxidants. J. Cereal Sci. 44(3): 236-251.

Emmons, C.L., and Peterson, D.M. (2001). Antioxidant activity and phenolic content of oat as affected by cultivar and location. Crop Sci. 41(6): 1676-1681.

Fares, C., Menga, V., Codianni, P., Russo, M., Perrone, D., Suriano, S., Savino, M., and Rascio, A. (2019). Phenolic acids variability and grain quality of organically and conventionally fertilised old wheats under a warm climate. J. Sci. Food Agric. 99(10): 4615-4623.

Food and Agriculture Organization. (2020). OECD-FAO Agricultural outlook 2020-2029. https://www.oecd-ilibrary.org/sites/57d27093-en/ index.html?itemld=/content/component/57d27093-en. Accessed 09/10/2021.

Food and Agriculture Organization. (2021). Post-harvesting process http://www.fao.org/3/au104e/au104e.pdf. Accessed 10 Sep. 2021.

$\mathrm{Fu}$, J., Soroka, D.N., Zhu, Y., and Sang, S. (2018). Induction of apoptosis and cell-cycle arrest in human colon-cancer cells by whole-grain alkyIresorcinols via activation of the p53 pathway. J. Agric. Food Chem. 66(45): 11935-11942.

Fu, J., Zhang, Y., Hu, Y., Zhao, G., Tang, Y., and Zou, L. (2020). Concise review: Coarse cereals exert multiple beneficial effects on human health. Food Chem. 325: 126761

Ge, X., Saleh, A.S., Jing, L., Zhao, K., Su, C., Zhang, B., Zhang, Q., and Li, W. (2021). Germination and drying induced changes in the composition and content of phenolic compounds in naked barley. J. Food Compos. Anal. 95: 103594

Giambanelli, E., Ferioli, F., and D’Antuono, L.F. (2018). Retention of alkylresorcinols, antioxidant activity and fatty acids following traditional hulled wheat processing. J. Cereal Sci. 79: 98-105.

Gong, L., Huang, L., and Zhang, Y. (2012). Effect of Steam Explosion Treatment on Barley Bran Phenolic Compounds and Antioxidant Capacity. J. Agric. Food Chem. 60(29): 7177-7184.

Horvat, D., Šimić, G., Drezner, G., Lalić, A., Ledenčan, T., Tucak, M., Plavsic, H., Andric, L., and Zdunić, Z. (2020). Phenolic acid profiles and antioxidant activity of major cereal crops. Antioxidants 9(6): 527.

Hu, Z., Tang, X., Liu, J., Zhu, Z., and Shao, Y. (2017). Effect of parboiling on phytochemical content, antioxidant activity and physicochemical properties of germinated red rice. Food Chem. 214: 285-292.

Irondi, E.A., Adegoke, B.M., Effion, E.S., Oyewo, S.O., Alamu, E.O., and Bo- 
ligon, A.A. (2019). Enzymes inhibitory property, antioxidant activity and phenolics profile of raw and roasted red sorghum grains in vitro. Food Sci. Hum. Wellness 8(2): 142-148.

Jonnala, R.S., Irmak, S., MacRitchie, F., and Bean, S.R. (2010). Phenolics in the bran of waxy wheat and triticale lines. J. Cereal Sci. 52(3): 509-515.

Koehler, P., and Wieser, H. (2013). Chemistry of cereal grains. Handbook on sourdough biotechnology. Springer, Boston, MA, pp. 11-45.

Kruk, J., Aboul-Enein, B., Bernstein, J., and Marchlewicz, M. (2017). Dietary alkylresorcinols and cancer prevention: A systematic review. Eur. Food Res. Technol. 243(10): 1693-1710.

Landberg, R., Kamal-Eldin, A., Salmenkallio-Marttila, M., Rouau, X., and Åman, P. (2008). Localization of alkylresorcinols in wheat, rye and barley kernels. J. Cereal Sci. 48(2): 401-406.

Li, W., Zhang, X., He, X., Li, F., Zhao, J., Yin, R., and Ming, J. (2020). Effects of steam explosion pretreatment on the composition and biological activities of tartary buckwheat bran phenolics. Food Funct. 11(5): 4648-4658.

Liu, J., Zhang, J., Wang, W., and Hou, H. (2021). Effects of microwave treatment on the stability and antioxidant capacity of a functional wheat bran. Food Sci. Nutr. 9(5): 2713-2721.

Liu, L., Zhao, M., Liu, X., Zhong, K., Tong, L., Zhou, X. and Zhou, S. (2016). Effect of steam explosion-assisted extraction on phenolic acid profiles and antioxidant properties of wheat bran. J. Sci. Food and Agr. 96(10): 3484-3491.

Liyana-Pathirana, C.M., and Shahidi, F. (2006). Importance of insolublebound phenolics to antioxidant properties of wheat. J. Agric. Food Chem. 54(4): 1256-1264.

Luo, M., Hou, F., Dong, L., Huang, F., Zhang, R., and Su, D. (2020). Comparison of microwave and high-pressure processing on bound phenolic composition and antioxidant activities of sorghum hull. Int. J. Food Science Technol. 55(9): 3190-3202.

Madhujith, T., and Shahidi, F. (2009). Antioxidant potential of barley as affected by alkaline hydrolysis and release of insoluble-bound phenolics. Food Chem. 117(4): 615-620.

Madhujith, T., Izydorczyk, M., and Shahidi, F. (2006). Antioxidant properties of pearled barley fractions. J. Agric. Food Chem. 54(9): 32833289.

Mishra, G., Joshi, D.C., and Panda, B.K. (2014). Popping and puffing of cereal grains: a review. J. Grain Processing Storage 1(2): 34-46.

Naczk, M., and Shahidi, F. (2006). Phenolics in cereals, fruits and vegetables: Occurrence, extraction and analysis. J. Pharm. Biomed. Anal. 41(5): 1523-1542.

Oboh, G., Ademiluyi, A.O., and Akindahunsi, A.A. (2010). The effect of roasting on the nutritional and antioxidant properties of yellow and white maize varieties. Int. J. Food Sci. 45(6): 1236-1242.

Pang, Y., Ahmed, S., Xu, Y., Beta, T., Zhu, Z., Shao, Y., and Bao, J. (2018). Bound phenolic compounds and antioxidant properties of whole grain and bran of white, red and black rice. Food Chem. 240: 212221.

Pielhop, T., Amgarten, J., Von Rohr, P.R., and Studer, M.H. (2016). Steam explosion pretreatment of softwood: The effect of the explosive decompression on enzymatic digestibility. Biotechnol. Biofuels 9(1): 152-152.

Pihlava, J.M., Hellström, J., Kurtelius, T., and Mattila, P. (2018). Flavonoids, anthocyanins, phenolamides, benzoxazinoids, lignans and alkylresorcinols in rye (Secale cereale) and some rye products. J. Cereal Sci. 79: 183-192.

Rabemanolontsoa, H., and Saka, S. (2016). Various pretreatments of lignocellulosics. Bioresour. Technol. 199: 83-91.

Raheem, D., Dayoub, M., Birech, R., and Nakiyemba, A. (2021). The Contribution of Cereal Grains to Food Security and Sustainability in Africa: Potential Application of UAV in Ghana, Nigeria, Uganda, and Namibia. Urban Sci. 5(1): 8.

Rao, S., Santhakumar, A.B., Chinkwo, K.A., and Blanchard, C.L. (2020). Investigation of phenolic compounds with antioxidant activity in barley and oats affected by variation in growing location. Cereal Chem. 97(4): 772-782.

Rempelos, L., Almuayrifi, A.M., Baranski, M., Tetard-Jones, C., Eyre, M., Shotton, P., Cakmak, I., Ozturk, L., Cooper, J., Volakakis, N., Schmidt, C., Sufar, E., Wang, J., Wilkinson, A., Rosa, E.A.S., Zhao, B., Rose, T.J.,
Leifert, C., and Bilsborrow, P. (2018). Effects of agronomic management and climate on leaf phenolic profiles, disease severity, and grain yield in organic and conventional wheat production systems. J. Agric. Food Chem. 66(40): 10369-10379.

Rodehutscord, M., Rückert, C., Maurer, H.P., Schenkel, H., Schipprack, W., Bach Knudsen, K.E., Schollenberger, M., Laux, M., Eklund, M., Siegert, W., and Mosenthin, R. (2016). Variation in chemical composition and physical characteristics of cereal grains from different genotypes. Arch. Anim. Nutr. 70(2): 87-107.

Rodríguez-García, C., Sánchez-Quesada, C., Toledo, E., Delgado-Rodríguez, M., and Gaforio, J.J. (2019). Naturally lignan-rich foods: A dietary tool for health promotion? Mol. 24(5): 917.

Ross, A.B., Kamal-Eldin, A., Lundin, E.A., Zhang, J.X., Hallmans, G., and Åman, P. (2003b). Cereal alkylresorcinols are absorbed by humans. J. Nutr. 133(7): 2222-2224.

Ross, A.B., Shepherd, M.J., Schüpphaus, M., Sinclair, V., Alfaro, B., KamalEldin, A., and Åman, P. (2003a). Alkylresorcinols in cereals and cereal products. J. Agric. Food Chem. 51(14): 4111-4118.

Schmidt, M., Zannini, E., and Arendt, E.K. (2018). Recent advances in physical post-harvest treatments for shelf-life extension of cereal crops. Foods 7(4): 45.

Serna-Saldivar, S.O. (2016). Cereal grains: properties, processing, and nutritional attributes. CRC press.

Setyaningsih, W., Hidayah, N., Saputro, I.E., Palma, M., and Barroso, C.G. (2016). Profile of phenolic compounds in Indonesian rice (Oryza sativa) varieties throughout post-harvest practices. J. Food Compos. Anal. 54: 55-62.

Shahidi, F. (2009). Nutraceuticals and functional foods: whole versus processed foods. Trends Food Sci. Technol. 20(9): 376-387.

Shahidi, F., and Naczk, M. (2003). Phenolics in food and nutraceuticals. CRC press.

Shahidi, F., and Yeo, J. (2016). Insoluble-bound phenolics in food. Mol. 21(9): 1216

Shahidi, F., Varatharajan, V., Oh, W.Y., and Peng, H. (2019). Phenolic compounds in agri-food by-products, their bioavailability and health ef fects. J. Food Bioact. 5(1): 57-119.

Shao, Y., Hu, Z., Yu, Y., Mou, R., Zhu, Z., and Beta, T. (2018). Phenolic acids, anthocyanins, proanthocyanidins, antioxidant activity, minerals and their correlations in non-pigmented, red, and black rice. Food Chem. 239: 733-741.

Sidhu, J.S., Kabir, Y., and Huffman, F.G. (2007). Functional foods from cereal grains. Int. J. Food Prop. 10(2): 231-244.

Singh, J., Suhag, M., and Dhaka, A. (2015). Augmented digestion of lignocellulose by steam explosion, acid and alkaline pretreatment methods: A review. Carbohydr. Polym. 117: 624-631.

Sripum, C., Kukreja, R.K., Charoenkiatkul, S., Kriengsinyos, W., and Suttisansanee, U. (2016). The effect of storage conditions on antioxidant activities and total phenolic contents of parboiled germinated brown rice (Khao Dok Mali 105). Int. Food Res. J. 23(4): 1827.

Statista. (2021). Worldwide production of grain in 2020/21, by type. https://www.statista.com/statistics/263977/world-grain-productionby-type/ Accessed 10 Sep. 2021.

Stumpf, B., Yan, F., and Honermeier, B. (2015). Nitrogen fertilization and maturity influence the phenolic concentration of wheat grain (Triticum aestivum). J. Soil Sci. 178(1): 118-125.

Sun, X.F., Xu, F., Sun, R.C., Geng, Z.C., Fowler, P., and Baird, M.S. (2005). Characteristics of degraded hemicellulosic polymers obtained from steam exploded wheat straw. Carbohydr. Polym. 60(1): 15-26.

Suriano, S., Balconi, C., Valoti, P., and Redaelli, R. (2021). Comparison of total polyphenols, profile anthocyanins, color analysis, carotenoids and tocols in pigmented maize. LWT 144: 111257.

Thammapat, P., Meeso, N., and Siriamornpun, S. (2016). Effects of the traditional method and an alternative parboiling process on the fatty acids, vitamin $E, \gamma$-oryzanol and phenolic acids of glutinous rice. Food Chem. 194: 230-236.

Vaher, M., Matso, K., Levandi, T., Helmja, K., and Kaljurand, M. (2010). Phenolic compounds and the antioxidant activity of the bran, flour and whole grain of different wheat varieties. Procedia Chem. 2(1): 76-82.

Wang, T., He, F., and Chen, G. (2014). Improving bioaccessibility and bioavailability of phenolic compounds in cereal grains through processing technologies: A concise review. J. Funct. Foods 7: 101-111. 
Wanyo, P., Meeso, N., and Siriamornpun, S. (2014). Effects of different treatments on the antioxidant properties and phenolic compounds of rice bran and rice husk. Food Chem. 157: 457-463.

Yu, S., and Tian, L. (2018). Breeding major cereal grains through the lens of nutrition sensitivity. Mol. Plant 11(1): 23-30.

Zanella, I., Biasiotto, G., Holm, F., and di Lorenzo, D. (2017). Cereal lignans, natural compounds of interest for human health? Nat. Prod. Commun. 12(1): 139-146.

Zeng, Z., Hu, X., McClements, D.J., Luo, S., Liu, C., Gong, E., and Huang, K. (2019). Hydrothermal stability of phenolic extracts of brown rice. Food Chem. 271: 114-121.

Zeng, Z., Liu, C., Luo, S., Chen, J., and Gong, E. (2016). The profile and bioaccessibility of phenolic compounds in cereals influenced by im- proved extrusion cooking treatment. PloS One 11(8): e0161086.

Zhang, Q., de Mejia, E.G., Luna-Vital, D., Tao, T., Chandrasekaran, S., Chatham, L., Juvik, J., Singh, V., and Kumar, D. (2019). Relationship of phenolic composition of selected purple maize (Zea mays L.) genotypes with their anti-inflammatory, anti-adipogenic and anti-diabetic potential. Food Chem. 289: 739-750.

Zhou, Z., Chen, X., Zhang, M., and Blanchard, C. (2014). Phenolics, flavonoids, proanthocyanidin and antioxidant activity of brown rice with different pericarp colors following storage. J. Stored Prod. Res. 59: 120-125

Živković, A., Polak, T., Cigić, B., and Požrl, T. (2021). Germinated Buckwheat: Effects of Dehulling on Phenolics Profile and Antioxidant Activity of Buckwheat Seeds. Foods 10(4): 740. 